\title{
A Fiftyfold Momentary Beam Intensification for a High-Voltage Cold-Cathode Oscillograph
}

\author{
John H. Park
}

\begin{abstract}
A method for obtaining a momentary increase (up to 50 times the steady value) in the intensity of the electron beam obtained from a high-voltage cold-cathode discharge tube has been developed. Its application for increasing the recording speed of a high-voltage cathoderay oscillograph is described. Oscillograms have been obtained in which the writing speed is about three-fourths the speed of light.

The intensification is caused by superposing a steeply rising voltage pulse on the normal steady voltage across the electrodes of the discharge tube serving as the electron beam source. The voltage pulse momentarily disrupts equilibrium conditions in the discharge and produces an intense discharge that lasts for about 2 microseconds. Measurements of the magnitude and duration of the superposed pulse and of the changes in discharge current have been made. A tentative explanation of the mechanism of intensification based on these measurements is given.
\end{abstract}

\section{Introduction}

High-voltage cold-cathode discharge tubes have long been used as sources of electron beams. One of their most important applications has been to supply the electron beam for high-speed cathode-ray oscillographs (CRO) used to record transient electric phenomena of very short duration. The establishment of a beam of high electron density is of paramount importance where very high "writing speeds" are required.

In the experimental work that will be described, the electron beam source ${ }^{2}$ was a 1-in.-diameter Pyrex glass tube provided with two cylindrical electrodes spaced 4 in. apart. The air pressure in the tube was adjustable and was maintained at approximately $10 \mu$ of mercury. With a direct voltage of $50 \mathrm{kv}$ applied between electrodes, a very narrow beam of visible discharge is obtained that originates in a bright spot at the center of the cathode and terminates at the anode. Since the mean free path for electrons in the discharge is approximately equal to the distance between electrodes, a large fraction of the electrons generated at the cathode are accelerated to a speed corresponding to full voltage between cathode and anode while travelling to the anode. A small hole in the center of the anode allows part of this electron beam to continue from the discharge tube into the main chamber of the $\mathrm{CRO}$ where the electrons pass through a beam trap, focusing coil, deflecting and sweep plates and finally strike the film or fluorescent viewing screen. The current in the discharge tube is very sensitive to small changes in air pressure. A fine control of pressure permits adjustment of the discharge current to values ranging from 0.1 to 0.8 ma. The portion of the electron beam passing through the hole in the anode and used for recording purposes is only

1 This investigation has been carried out as part of a cooperative program on basic instrumentation jointly sponsored at the National Bureau of Standards by the Office of Naval Research, the Office of Air Research, and the Atomic Energy Commission.

${ }^{2}$ A Westinghouse $50-\mathrm{kv}$ cathode-ray oscillograph (with minor modifications) was used in all the experimental work. about 1 percent of the total discharge-tube current.

In order to obtain useable records of electrical transients of very short duration, that is, high "writing speeds", a high electron density in the beam is required. Some of the various methods ${ }^{3456}$ that have been used to increase beam intensity are (1) shaping the electrodes, (2) using different gases in the discharge tube, and (3) using prefocusing coils placed around the discharge tube. Also the fine control of gas pressure that permits adjustment of current in the discharge tube can be used to increase beam intensity up to a certain point; but any attempt to establish a steady discharge current above approximately $0.8 \mathrm{ma}$ destroys equilibrium and changes the type of discharge so that a steady beam cannot be maintained. These methods of intensification have proved moderately successful. However, it was found that by superposing a momentary pulse on the constant direct-current voltage applied to the discharge tube, the discharge current and beam intensity could be momentarily increased by a factor of 10 to 50 times the steady state value. The increased beam current persists only for a very short time and does not appreciably decrease the life of the cathode, but by proper synchronization with the phenomena being measured the increased beam intensity becomes useful for obtaining higher writing speeds.

\section{Experimental Methods and Measurements}

A momentary increase in current through the discharge tube and beam intensification occurs whenever a sudden increase of about $800 \mathrm{v}$ or more is superposed on the normal steady value of $50 \mathrm{kv}$ applied between the cathode and anode. The following methods of applying this pulse have been

${ }^{3}$ W. Rogowski, Limitations of cathode-ray oscillograph, Elektrotech. Z. 52 1245 (Oct. 1931).

${ }^{4}$ F. Malsch, Electron current density in CRO, Arch. Elektrotech. 27, 642 Sept. 1933)

5 J. M. Bryant and M. Newman, Developments in hig' speed cathode ray oscillography, Univ. Minn. Engr. Expt. Sta. Tech. Pap. 27 (March 1942).

${ }_{6}$ E. T. Wade, T. J. Carpenter, and D. D. MacCarthy, Modern cathode-ray oscillograph for testing lightning arresters, Trans. AIEE 61. 549 (May 1942). 


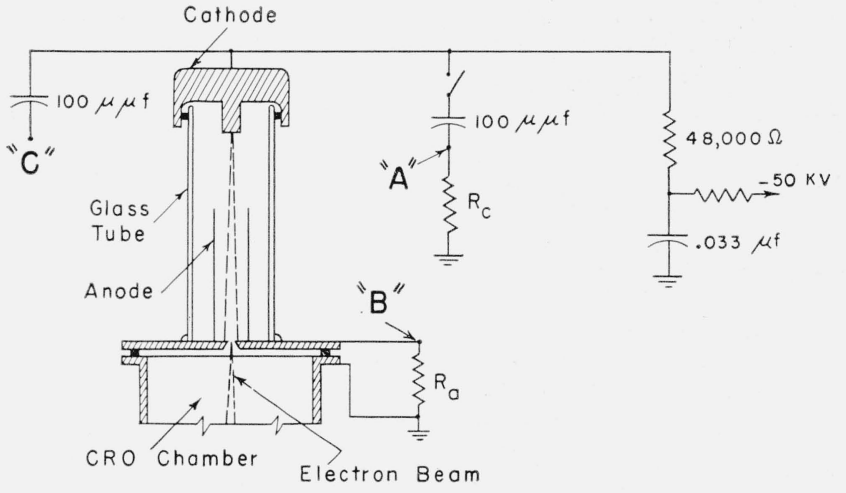

FiguRE 1. Wiring diagram of circuits used for (1) applying the intensifying pulse to the discharge tube supply voltage and (2) obtaining measurements of this pulse and changes in current through the discharge tube.

tried and all give the intensification: (1) A current pulse passed through a resistor between anode and ground return lead. (2) A voltage pulse capacitively coupled with the cathode. (3) A current pulse inductively coupled with the lead to the cathode or anode. Because of the difficulties encountered in the control and measurement of the applied voltage pulse for method (3), a detailed description of procedure and results will be given only for methods (1) and (2).

\subsection{Current Pulse through Anode Resistor}

Resistor $R_{a}$ and connections at $B$ and $C$ (see fig. 1) were used for this method, and the single-pole switch just above $A$ was open. The anode of the discharge tube was insulated from the grounded case of the CRO by removing the screws that clamp the rubber gasket separating the discharge chamber from the main CRO case. The anode was connected to ground through resistor $R_{a}$. By passing a current pulse through $R_{a}$ the potential difference between anode and cathode could be given a similar pulse. The magnitude and wave shape of this pulse were measured by connecting $B$ to the CRO deflecting plates. By connecting $C$ to the deflecting plates and thus using the deflecting plates as the low side of a capacitance divider, a record of change in potential of the cathode was also obtained. This record of the change in cathode potential when divided by 48,000 is also a record of the change in current through the discharge tube, since for the short duration of the pulse the voltage of the cathode can only be changed by the same amount as the IR drop through the 48,000 -ohm resistor, which is in series between the cathode and the $0.033-\mu$ f smoothing capacitor.

By connecting $B$ to various points on the resistance network that supplies the Norinder ${ }^{7}$ relay voltages for the CRO, the magnitude, duration, and polarity of $E_{a}$ (voltage pulse applied to the anode) could be controlled. Records were obtained for various conditions, and two examples are shown in figure 2 . The horizontal trace, nearest the time scale in each

${ }^{7}$ O. Ackermann, A cathode ray oscillograph with Norinder relay, Trans. AIEE 39, 467 (April 1930). oscillogram, is the reference "zero line" obtained with the deflecting plates short-circuited. The voltage applied to the anode, $E_{a}$, rises to a maximum very rapidly ( $<0.5 \mu \mathrm{sec})$ and decreases fairly slowly. The current, $i_{d}$, through the discharge tube increases from its low steady-state value of $0.1 \mathrm{ma}$ to a fairly high value, approximately $7 \mathrm{ma}$, as soon as the anode voltage is applied. When the polarity of the anode pulse is positive (fig. 2, A), it increases the voltage between anode and cathode and a short time (about $1 \mu \mathrm{sec}$ ) after application of the anode pulse (this delay time being shorter for higher anode voltages and for higher values of initial discharge current) the discharge current shows a second rapid rise and the intensity of the electron beam increases at the same time. As the discharge current decreases, the beam intensity also decreases. When the polarity of the anode pulse is negative (fig. 2, B), it decreases the voltage between anode and cathode. The net current in the discharge tube is temporarily reversed from its steady-state direction and is of a greatly increased magnitude. Nevertheless, a beam of electrons continues to flow in the original direction and is even somewhat intensified.

\subsection{Voltage Pulse Capacitively Coupled With Cathode}

For this method the single pole switch above $A$ (see fig. 1) was closed, thus connecting the cathode to one terminal of a small high-voltage capacitor (actually consisting of a polyethylene-insulated wire with a braided sheath over the insulation). The other terminal of this capacitor was connected to ground through resistor $R_{c}$. With the steady direct-current voltage on the cathode this capacitor is charged to $50 \mathrm{kv}$, and when a current pulse is passed through $R_{c}$ the cathode voltage is immediately changed by the drop through $R_{c}$. The duration of this change in cathode voltage is not fixed by the duration of the current pulse through $R_{c}$ but is determined by the time it takes for the small highvoltage capacitor to assume a new value of charge, that is, by the time constant of the small capacitance in series with the $48,000-0 h m$ resistor (the effective resistance of the discharge tube being much larger). A record of this change in cathode voltage was obtained by connecting $C$ to the CRO deflecting plates. A record of the current through the discharge tube was obtained by making $R_{a} 100,000 \mathrm{ohms}$ and connecting $B$ to the CRO deflecting plates.

By connecting $A$ to various points on the resistance network that supplies the Norinder relay voltages for the CRO, the magnitude and polarity of $E_{c}$ (change in cathode voltage) could be controlled. Records were obtained for various conditions, and two examples are shown in figure 3 . With a negative pulse applied to the cathode (increase in voltage between cathode and anode) the discharge current increases to a high value immediately. Beam intensification and a second increase in discharge current occur a short time later. In the example shown in figure $3, \mathrm{~A}$, the magnitude of the applied 

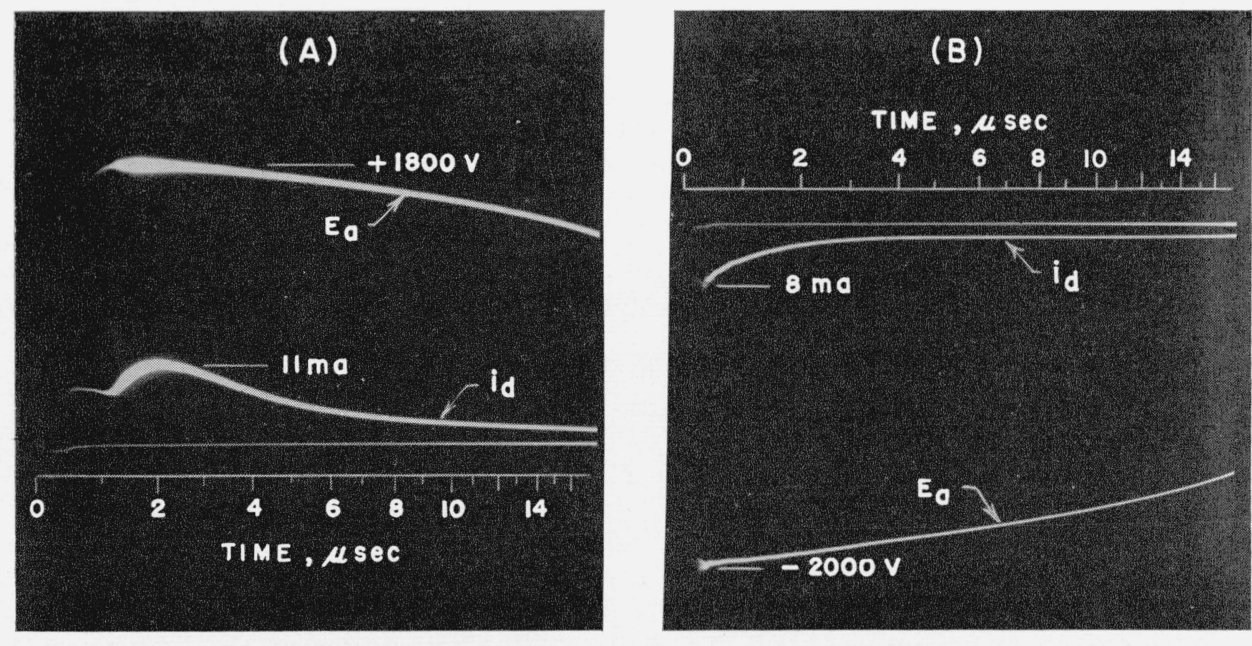

Figure 2. Oscillograms of voltage pulses applied to the anode, $E_{a}$, and the corresponding changes in current through the discharge tube, $i_{d}$.

(A) is for a positive polarity voltage pulse, and (B) is for a negative polarity voltage pulse.
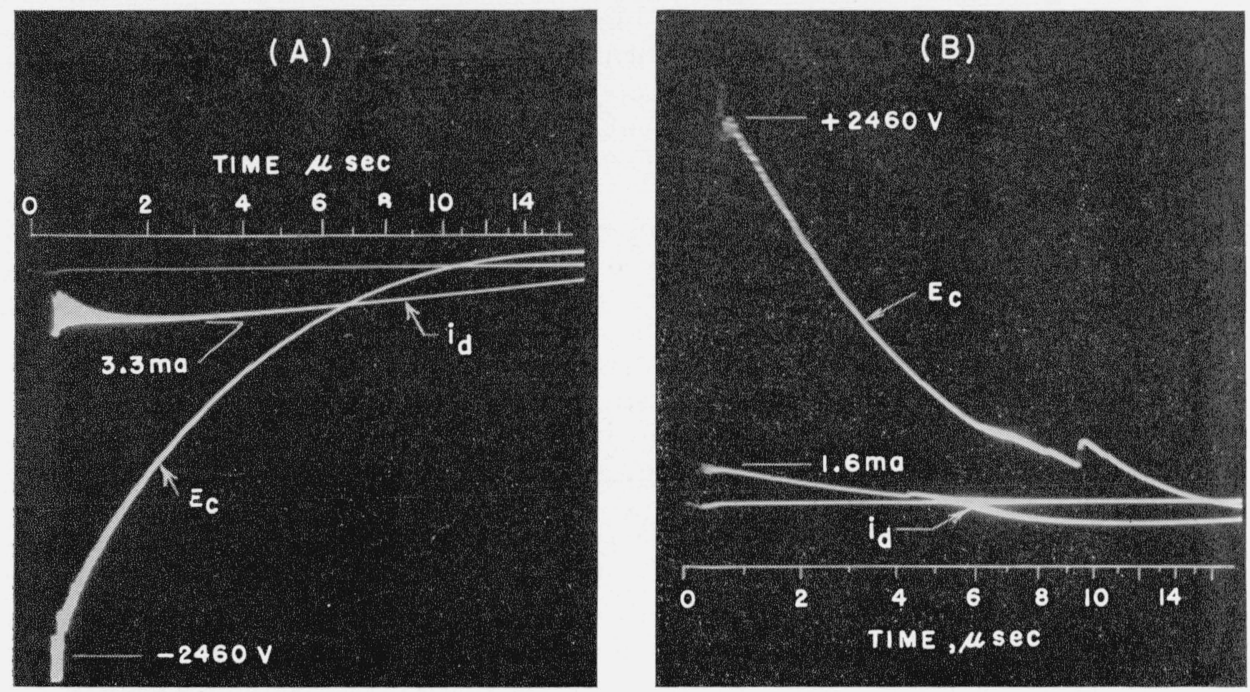

Figure 3. Oscillograms of voltage pulses applied to the cathode, $E_{c}$, and the corresponding changes in current through the discharge tube, $i_{d}$.

(A) is for a negative polarity voltage pulse, and (B) is for a positive polarity voltage pulse.

cathode pulse was so high that beam intensification and second increase in discharge current occur very quickly. The discharge current remains high even after the cathode voltage pulse has decreased to zero. Records with slower sweeps indicate that the discharge current and beam intensity continue to decrease and at a somewhat later time (approximately $200 \mu \mathrm{sec}$ ) the beam fails to leave a trace on the film. A momentary blink in the visible discharge can be observed when the cathode-anode voltage is suddenly increased by a large amount ( $2 \mathrm{kv}$ or more). With a positive pulse applied to the cathode (decrease in voltage between cathode and anode) the discharge current immediately increases to a high value, reversed in direction from the steady-state discharge current. This current decreases as the cathode pulse voltage decreases, and unless the maximum value of the cathode pulse is above approximately $1,000 \mathrm{v}$, very little beam intensification occurs. For the example shown in figure $3, \mathrm{~B}$, the cathode pulse was above this minimum value and beam intensification, and reversal of discharge current (to the normal steady state direction) occur about $5 \mu$ sec after cathode pulse is initiated. Oscillograms taken under other conditions indicate that this time delay decreases as the magnitude of the cathode pulse is increased and as the initial steady-state discharge current is increased.

\section{Proposed Explanation of Experimental Measurements}

The reversal in direction of current through the discharge tube (i. e., reversed from the normal steady-state condition) as shown in figures 2, B and $3, \mathrm{~B}$, indicates that for short times, energy can be taken from the discharge without appreciably affecting the mechanism of beam generation, since the electron beam density remains nearly constant as shown by constant density in the trace on the oscillogram. Also, as shown in figure 2, A, with an applied pulse that increases cathode-anode voltage, energy can be fed into the discharge during the short period before beam intensification starts without affecting the recording electron beam. In order to explain these results, consider the variation of net space charge density in the discharge between 
cathode and anode. There is a high concentration of both electrons and positive ions throughout the volume of the discharge. Electrons are being constantly fed into the region near the cathode, and since it takes a significant, even though short, time for them to be accelerated toward the anode by the impressed electric field, a large net negative space charge region is built up near the cathode. In the region near the anode, including the space inside the tube forming part of the anode, gas molecules are continually being ionized by the short-wave radiation produced by the impact of high-speed electrons on the anode. The slow electrons produced in this manner drift toward the anode and leave an excess of positive ions, which tend to be accelerated toward the cathode but in the meanwhile build up a high net positive space-charge region near the anode. These space-charge regions have very marked effects on the current fed into the discharge tube, especially during sudden changes in applied voltage. However, the current in the discharge is probably mainly determined by (1) the number of positive ions produced by the short-wave radiation caused by each high-speed electron reaching the anode, and (2) the number of electrons generated at the cathode by each of these positive ions. This process is dependent on gas density and makes the steady-state discharge current so sensitive to gas pressure.

Under steady-state conditions, with $50-\mathrm{kv}$ direct current applied between cathode and anode and with the pressure in the discharge tube adjusted so that a normal electron beam is obtained $(0.1$ to $0.5 \mathrm{ma}$ in the discharge), it may be assumed that the high concentration of negative space charge in the region near the cathode makes the gradient at the cathode quite small in comparison to the average gradient between electrodes. Similarly, the gradient near the anode should be quite small. When the voltage between cathode and anode is suddenly changed, an instantaneous change in charge distribution can only occur on the electrode surfaces. Since the electrodes more nearly approximate points than infinite planes, the sudden change in their surface charge causes a sudden increase of gradient, mainly in the regions near the electrodes. The sudden increase in gradient causes electrons or positive ions to be drawn from each space charge region toward the adjacent electrode. This flow of electrons and ions constitutes a current flow in the external supply circuit, which is superposed on the steady-state current in the discharge tube. If there is a sudden decrease in cathode-anode voltage, there will be a net flow of electrons from the cathode space-charge region to the cathode and a net flow of positive ions from the anode space-charge region to the anode; these constitute the "reversed" current illustrated in figure 2 , B, which continues until the gradients near the electrodes are reduced to their normal low values.

If there is a sudden increase in the cathode-anode voltage, electrons and ions will flow in the direction to increase the negative space-charge density in the cathode region and to increase the positive space- charge density in the anode region. This is illustrated by the first part of the $i_{d}$ trace in figure $2, \mathrm{~A}$. A sudden increase of available electrons in the space near the cathode, if it is of sufficient magnitude, will disrupt the equilibrium conditions existing in the discharge as shown in figure 2, A, by the second increase in discharge current accompanied by beam intensification. A possible explanation of this sudden change in equilibrium conditions in the discharge is as follows: The increase of negative space charge in the region near the cathode causes an increase (probably gradual at first) in magnitude of the electron stream from cathode space-charge region to anode space-charge region. The greater the number of fast electrons reaching the anode the greater will be the intensity of the short-wave radiation that increases the supply of positive ions; and they will be accelerated toward the cathode and produce more electrons. Thus the action is cumulative. If the initial change is small or occurs at a low rate, a new equilibrium condition will be attained with little effect on the electron beam. If the initial disturbance is of sufficient magnitude and occurs suddenly the cumulative effect causes beam intensity and discharge current to increase rapidly (see fig. 2 , A). This effect is stopped very soon because the number of positive ions that can be produced in this short time is limited by the low gas pressure (total number of gaseous molecules) in the tube. As the number of available positive ions is reduced, the beam intensity and discharge current gradually decrease and in some cases the discharge is momentarily extinguished.

When the sudden change in voltage between cathode and anode is a pulse of short duration, as illustrated in figure 3 , the effects and their explanation are quite similar with one exception - that beam intensification as shown in figure $3, \mathrm{~B}$, is obtained when the polarity of the voltage pulse is such as to reduce the cathode-anode voltage. Note here that the high intensification does not occur until the superposed voltage pulse has decreased to about one-sixth its peak value and that before this time the gradients at the electrodes have been in the direction to decrease the magnitude of total space charge in each space-charged region (as indicated by the reversed direction of discharge current). Intensification occurs as the cathode-anode voltage returns to its normal value, which for the reduced magnitudes of space charge at this instant is sufficient to cause high gradients near the electrodes in the direction to increase the magnitude of charge in the space-charge regions. Thus, the physical explanation for beam intensification and increase in discharge current is the same as that given above, except that the method of initiation is different. As already noted, the high value of discharge current shown in figure $3, \mathrm{~A}$, persists even after the intensification has subsided and the disturbing voltage pulse has reached zero. This is additional evidence that the beam intensification and "second" increase in discharge current are caused by a "triggering" action that is initiated by the sudden increase in 
negative space charge near the cathode.

Some beam intensification may be observed in figure $3, \mathrm{~B}$, as soon as the voltage pulse is applied (the direction of the pulse being such as to decrease the cathode-anode voltage). This minor intensification might be accounted for by a concentrating effect on the beam caused by a temporary change in the shape of the electric field near the electrodes when the voltage between cathode and anode is suddenly changed.

\section{Application to Cathode-Ray Oscillographs}

The main reason for investigating the phenomena of momentary beam intensification was that such a study might lead to increased writing speed for the high-voltage CRO. The extremely high beam intensification lasts for about $2 \mu$ sec (see figs. 2 and 3 ) and can be made to come to full intensity within $0.25 \mu \mathrm{sec}$ after initiation of the intensifying pulse. Oscillographs now available give satisfactory records for sweep times of $1 \mu \mathrm{sec}$ or more. Improvement of writing speed is desired mainly for sweep times of less than 1 usec. Thus the beam intensification is of sufficient duration provided it can be accurately synchronized with the CRO sweep.

The CRO employed in these beam-intensification studies uses pulses to supply the Norinder relay or beam unblocking plates and sweep plates. These pulses are obtained by discharging capacitors, charged to $3,000 \mathrm{v}$, through resistance or resistancecapacitance circuits as indicated in figure 4 . The resistance circuit provided to supply voltages to the Norinder relay plates (used to unblock the beam for the short time required for one sweep of the beam

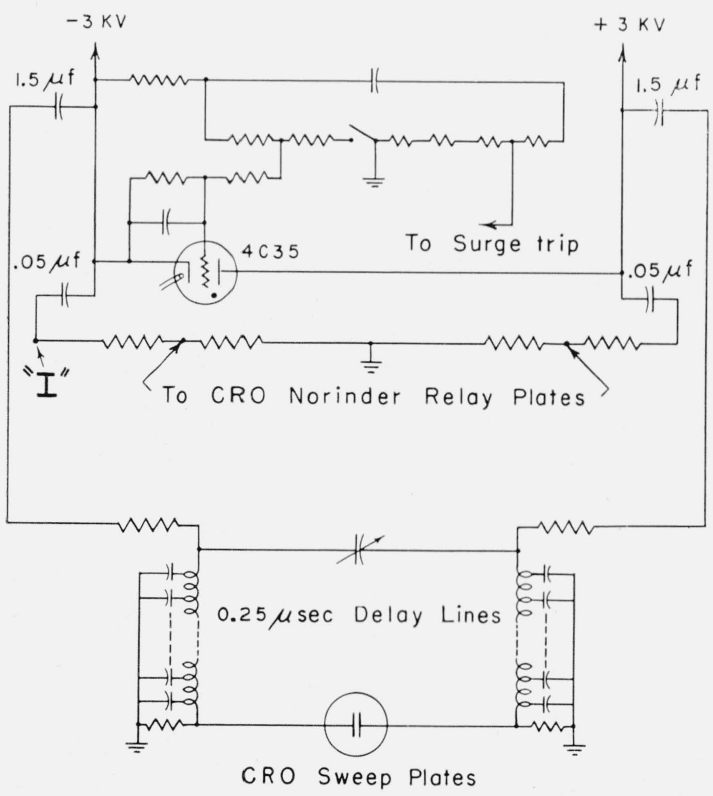

Figure 4. Wiring diagram of circuits used to supply the voltage surges for the Norinder relay plates and sweep plates of the CRO.

Intensifying pulse was obtained by connecting to point $I$. across the film) may be conveniently used to supply the intensifying pulse. It is only necessary to connect from point $I$ on this circuit to the sheath of the high-voltage (polyethylene-insulated) lead to the cathode. This connection gives positive synchronization of the intensification with the sweep, but since the time to attain maximum intensification is about $0.25 \mu \mathrm{sec}$, shorter sweep times would show no intensification. This timing discrepancy is compensated for by adding 0.25 - $\mu$ sec delay lines in the supply to the sweep plates as indicated in figure 4 . This delay in sweep supply has the added advantage of allowing the voltage on the Norinder relay plates to reach a high uniformly changing value before the sweep is started, which is quite important for very short sweep times.

Examples of the records obtained by using the intensifying pulse on very fast sweeps (60 in./ $\mu \mathrm{sec})$ are shown in figures $5^{8}$ and 6 . Without the intensifying pulse the only record visible is a very faint zero line. The oscillogram shown in figure 5 was obtained by coupling the CRO deflecting plates with as short a loop as possible directly to the plate circuit of a 150-megacycle oscillator consisting of two 6J6 tubes in push-pull parallel. The deflection shown in figure 5 corresponds to $500 \mathrm{v}$ rms on the deflecting plates, which was obtained by momentarily pulsing the plate supply of the oscillator to $1,200 \mathrm{v}$. This momentary high plate voltage was used not only in order to attain the high momentary output from the oscillator but also to prevent breakdown between the deflecting plates inside the CRO. It was found that a steady radio frequency as low as $100 \mathrm{v}$ rms applied to the deflecting plates caused a gaseous discharge to appear between plates when

${ }^{8}$ As seen from these records, the sweep speed is neither exactly uniform nor logarithmic. The slight packing of the time scale on the initial part of the sweep is introduced by the $0.25-\mu$ sec 10 -section delay lines inserted in the sweep leads.

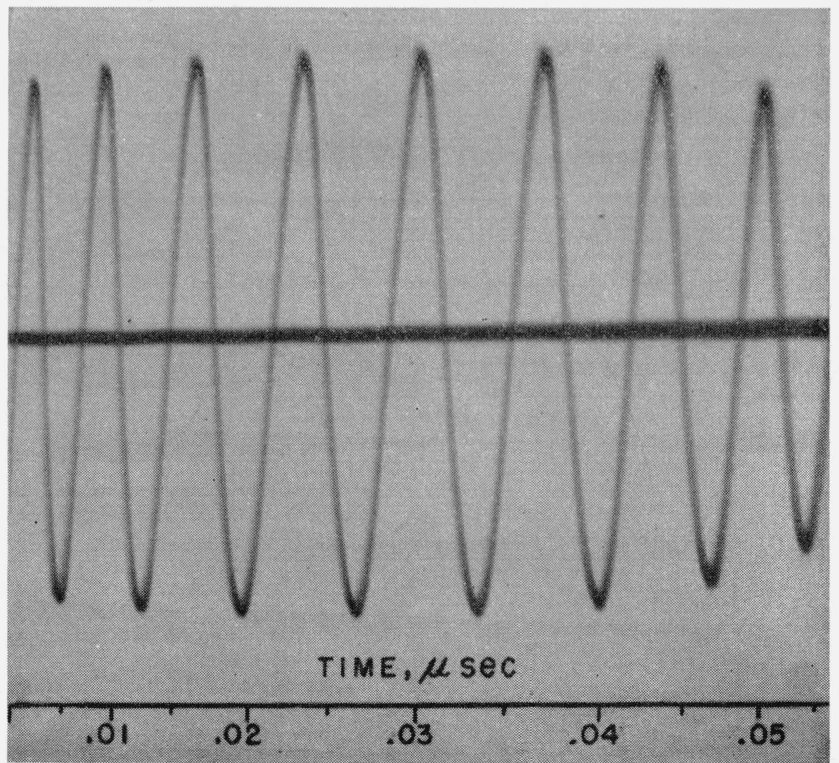

FiguRE 5. Oscillogram of the voltage wave-form obtained from a 150-megacycle pulsed oscillator.

The intensified beam was used to obtain this record. 
the beam is unblocked. No discharge appears with direct-current or pulse voltages, whose magnitudes are $2,000 \mathrm{v}$ or higher.

The oscillogram shown in figure 6 is one of a number taken in an attempt to determine the maximum writing speed attainable with the intensified beam. The deflecting plates were connected to a small neon tube lightning arrester (intended for use on telephone circuits), using the shortest leads possible. A steeply rising voltage pulse was applied to the arrester tube by connecting it across a 500-ohm resistor through which a capacitor was discharged. Figure 6 shows the initial part of the rise of this voltage. Breakdown of the arrester occurs after the voltage has gone off scale on the oscillogram. The subsequent oscillations shown on the record are produced when the deflecting plates are short-circuited by the arrester discharge, after being charged to a high voltage (about $6,000 \mathrm{v})$. This high value of charging voltage, which caused the initial rise of voltage and the first four oscillation peaks to go off scale on the oscillogram, was used in order to attain as high a rate of change of voltage on the deflecting plates as possible. As seen from the record, the beam intensity is sufficient to obtain a visible trace even with this high rate of change of voltage. The trace on the oscillogram is a record of the change of voltage with time and it is very nearly perpendicular to the time axis. Since a trace made on the film, with voltage on the deflecting plates only, was found to be not exactly perpendicular to the zero line, measurements of time between maximum deflection in one direction and maximum deflection in the opposite direction are not significant. This is accounted for by the fact that in order to have the record start at the left edge of the film the beam is deflected off the axis of the CRO by deflecting coils just before it passes through the deflecting plates, and this makes the beam travel through an unsymmetrical fringing field just as it leaves the deflecting plates. In order to obtain writing speeds corrected for this distortion, the time between the two vertical traces of a positive or negative half cycle whose peak was off the film was measured (1) at the zero line and (2) at the place where the trace just goes off the film. The difference between these two measured times is the time it takes the beam to travel from the zero line to the off-scale point and back to the zero line (a distance of $5 \frac{1 / 2}{2}$ in. on the film). Measurements of writing speed made by this method on the record reproduced in figure 6 , and 10 other similar film records give an average writing speed for the trace appearing on the film during the first cycle of the oscillations of 9,100 in./ $\mu$ sec (about three-fourths the speed of light).

The value of writing speed given above is for a trace that is easily visible on the film, the maximum writing speed being fixed by (1) the maximum rate of change of deflection that can be imparted to the electron beam while traveling between the deflecting plates, and (2) the distance from the plates to the recording film. This maximum rate of change of deflection is limited by (1) the time of travel of the electrons of the beam through the deflecting plate

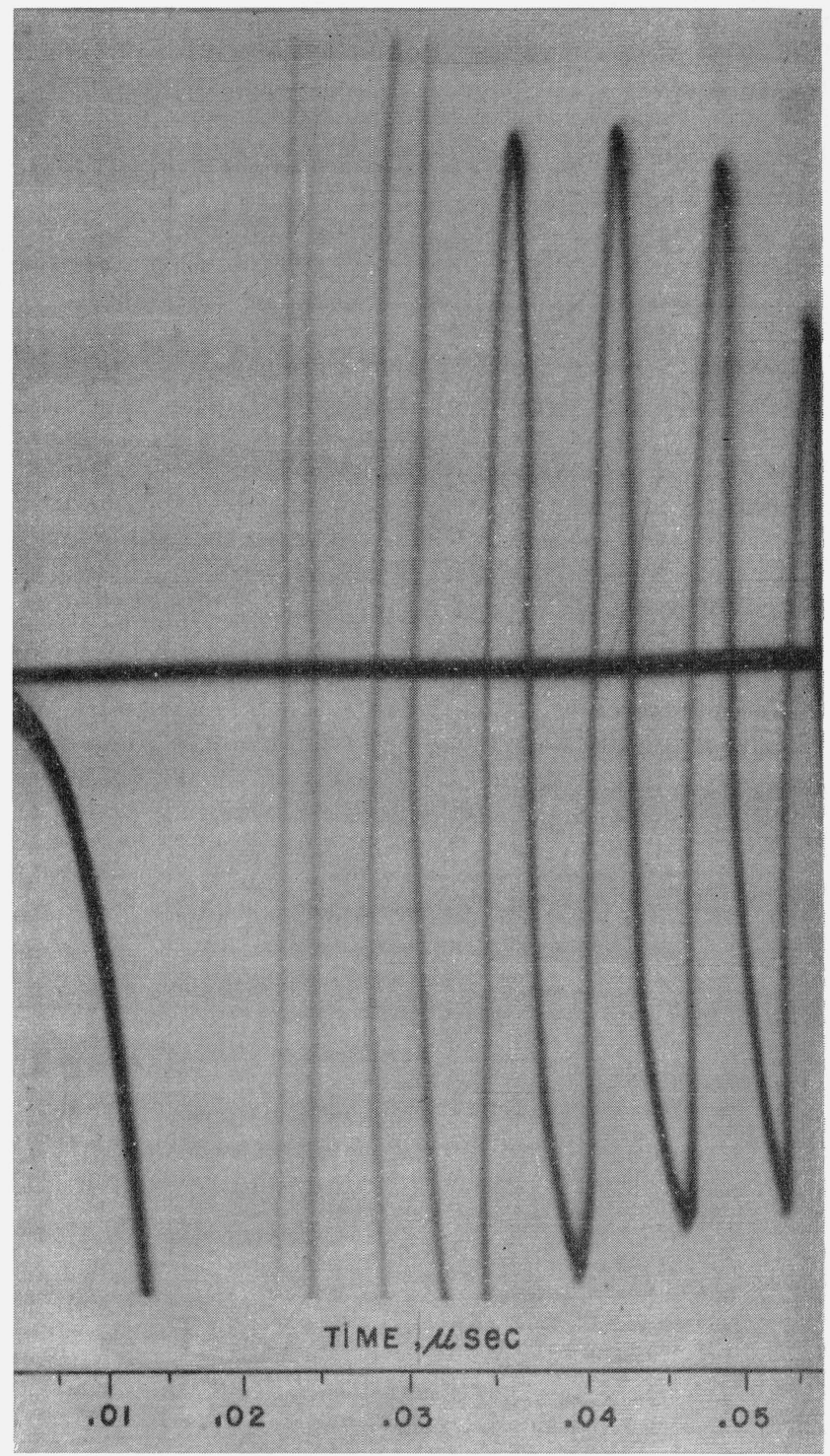

Figure 6. Oscillogram of the oscillations obtained when the CRO deflecting plates are short-circuited after being charged to 6,000 volts.

The maximum speed of the recording trace on this film was about 9,100 inches per microsecond.

field, and (2) the maximum rate of change of the electric field between the deflecting plates. The maximum rate of change of voltage between deflecting plates is attained when the plates are charged to the highest voltage possible and then discharged through the shortest leads possible. These conditions were fulfilled as nearly as possible in obtaining the record shown in figure 6 . A computation of this rate of change of voltage was made by using (1) the measured value of capacitance between plates (10 $\mu \mu f),(2)$ an estimated effective length of leads between the plates and the point at which these leads were short-circuited by the small neon tube arrester (8 in.), and (3) a maximum charging voltage on the plates of $6,000 \mathrm{v}$. Using this computed rate of 
change of voltage and taking into account the time of travel of electrons through the deflecting plate field and the distance between the deflecting plates and the recording film, a computed value of maximum writing speed for the CRO used in the experiments described above was found to be $9,700 \mathrm{in}$./ $\mu \mathrm{sec}$.

\section{Conclusions}

The computed and experimental values of maximum writing speed agree quite well, and as already noted this maximum value is determined by the design of the CRO used in these experiments rather than the intensity of the electron beam. The electron beam intensity as used in the experiments is probably sufficient to yield writing speeds greater than the speed of light if a CRO deflecting system, designed with this objective in mind, were used.

Thus the method of beam intensification described in this paper can be used to obtain extremely high writing speeds, and it can very easily be applied to a high-voltage cold-cathode CRO. Also, since the current in the discharge tube is maintained at a low steady value except for the very short time required to obtain the record, the problems of beam adjustment and life of cathode become of minor importance. It should be noted that the intensifying pulse increases the electron beam voltage slightly (about 2 to $5 \%$, depending on the magnitude of the pulse being used). This decreases the voltage sensitivity of the CRO by the same percentage and suggests that a voltage calibration be made when the intensifying pulse is used if a higher percentage accuracy is desired from the CRO records.

In addition to its use for increasing the writing speed of a high-voltage CRO, the method described should be useful wherever a high-density electron beam of short duration (about $2 \mu$ sec.) is required.

Washington, February 15, 1951. 